\title{
On the Asymptotic Behavior of Positive Solutions of Certain Fractional Differential Equations
}

\author{
Said R. Grace \\ Department of Engineering Mathematics, Faculty of Engineering, Cairo University, Orman, Giza 12613, Egypt \\ Correspondence should be addressed to Said R. Grace; saidgrace@yahoo.com \\ Received 8 July 2015; Accepted 25 August 2015 \\ Academic Editor: Zhen-Lai Han \\ Copyright (C) 2015 Said R. Grace. This is an open access article distributed under the Creative Commons Attribution License, which \\ permits unrestricted use, distribution, and reproduction in any medium, provided the original work is properly cited. \\ This paper deals with the asymptotic behavior of positive solutions of certain forced fractional differential equations of the form \\ ${ }^{C} D_{c}^{\alpha} y(t)=e(t)+f(t, x(t)), c>1, \alpha \in(0,1)$, where $y(t)=\left(a(t) x^{\prime}(t)\right)^{\prime}, c_{0}=y(c) / \Gamma(1)=y(c)$, and $c_{0}$ is a real constant. From the \\ obtained results, we derive a technique which can be applied to some related fractional differential equations.
}

\section{Introduction}

Consider the forced fractional differential equation

$$
{ }^{C} D_{c}^{\alpha} y(t)=e(t)+f(t, x(t)), \quad c>1, \alpha \in(0,1),
$$

where $y(t)=\left(a(t) x^{\prime}(t)\right)^{\prime}, c_{0}=y(c) / \Gamma(1)=y(c)$, and $c_{0}$ is a real constant.

In the sequel we assume that

(i) $a:[c, \infty) \rightarrow R^{+}=(0, \infty)$ is a continuous function;

(ii) $f:[c, \infty) \times R \rightarrow R$ is continuous and assume that there exists a continuous function;

$h:[c, \infty) \rightarrow(0, \infty)$ and a real number, $\lambda, 0<\lambda \leq 1$ and $\gamma$ is a real number such that

$$
0 \leq x f(t, x) \leq t^{\gamma-1} h(t)|x|^{\lambda+1} \quad \text { for } x \neq 0, t \geq c \text {. }
$$

We only consider those solutions of (1) that are continuable and nontrivial in any neighborhood of $\infty$. Such solution is said to be oscillatory if there exists a sequence $\left\{t_{n}\right\} \subseteq[c, \infty)$, $t_{n} \rightarrow \infty$ such that $x\left(t_{n}\right)=0$, and it is nonoscillatory otherwise.

In the last few decades, integral, integrodifferential, and fractional differential equations have gained considerably more attention due to their applications in many engineering and scientific disciplines as the mathematical models for systems and processes in fields of physics, mechanics, chemistry, aerodynamics, and the electrodynamics of complex media. For more details one can refer to [1-8].

Oscillation and asymptotic behavior results for integral, integrodifferential, and fractional differential equations are scarce; some results can be found in [5, 9-13]. It seems that there are no such results for forced fractional differential equations of type (1). The main objective of this paper is to establish some new criteria on the asymptotic behavior of positive solutions of (1) which is equivalent to the Volterra type equation:

$$
\begin{array}{r}
y(t)=c_{0}+\frac{1}{\Gamma(\alpha)} \int_{c}^{t}(t-s)^{\alpha-1}[e(s)+f(s, x(s))] d s, \\
c>1, \alpha>0 .
\end{array}
$$

From the obtained results, we derive a technique which can be applied to some related fractional differential equations.

We note that

$$
{ }^{c} D_{a}^{\alpha} x(t):=\frac{1}{\Gamma(n-\alpha)} \int_{a}^{t}(t-s)^{n-\alpha-1} x^{(n)}(s) d s
$$

is the Caputo derivative of the order $\alpha \in(n-1, n)$ of a $C$-scalar valued function $x(t)$ defined on the interval $[c, \infty), x^{(n)}(t)=$ $d^{n} x(t) / d t^{n}$. For the case when $\alpha \in(n-1, n)$, this definition has been given by Caputo [14]. For the definition of the Caputo derivative of order $\alpha \in(n-1, n), n \geq 1$, see $[1,15,16]$. 


\section{Main Results}

To obtain our main results of this paper, we need the following two lemmas.

Lemma 1 (see $[5,7])$. Let $\beta, \gamma$, and $p$ be positive constants such that $[p(\beta-1)+1]>0, p(\beta-1)+1>0$. Then

$$
\int_{0}^{t}(t-s)^{p(\beta-1)} s^{p(\gamma-1)} d s=t^{\theta} B, \quad t \geq 0,
$$

where $B:=B[p(\gamma-1)+1, p(\beta-1)+1], B[\xi, \eta]=\int_{0}^{1} s^{\xi-1}(1-$ $s)^{\eta-1} d s,(\xi>0, \eta>0)$, and $\theta=p(\beta+\gamma-2)+1$.

Lemma 2 (see [17]). If $X$ and $Y$ are nonnegative, then

$$
X^{\lambda}-(1-\lambda) Y^{\lambda}-\lambda X Y^{\lambda-1} \leq 0, \quad 0<\lambda<1,
$$

where equality hold if and only if $X=Y$.

In what follows, we let

$$
\begin{aligned}
& g(t)=\frac{\left((1-\lambda) \lambda^{\lambda /(1-\lambda)}\right)}{\Gamma(\alpha)} \\
& \cdot \int_{t_{1}}^{t}(t-s)^{\alpha-1}\left(s^{\gamma-1} m^{\lambda /(\lambda-1)}(s) h^{1 /(1-\lambda)}(s)\right) d s
\end{aligned}
$$

and $0<\lambda<1, t \geq t_{1}$ for some $t_{1} \geq c$, where $m:[c, \infty) \rightarrow$ $(0, \infty)$ is a continuous function.

Now we give sufficient conditions under which any solution $x$ of (1) satisfies $x(t)=O\left(t^{2}\right)$ as $t \rightarrow \infty$.

Theorem 3. Let $0<\lambda<1$ and suppose that $p>1, q=$ $p /(p-1), 0<\alpha<1$ and $\gamma=2-\alpha-1 / p, p(\alpha-1)+1>0$, $p(\gamma-1)+1>0$, and $t / a(t)$ is bounded on $[c, \infty)$,

$$
\begin{aligned}
& \int_{t_{1}}^{\infty} \frac{s}{a(s)} d s<\infty, \\
& \int_{t_{1}}^{\infty}\left(s^{2} m(s)\right)^{q} d s<\infty,
\end{aligned}
$$

$$
\limsup _{t \rightarrow \infty} \frac{1}{t^{2}} \int_{c}^{t} \frac{1}{a(v)} \int_{t_{1}}^{v} \int_{c}^{u}(u-s)^{\alpha-1} e(s) d s d u d v
$$$$
<\infty \text {, }
$$

$$
\liminf _{t \rightarrow \infty} \frac{1}{t^{2}} \int_{c}^{t} \frac{1}{a(v)} \int_{t_{1}}^{v} \int_{c}^{u}(u-s)^{\alpha-1} e(s) d s d u d v
$$

$$
>-\infty
$$$$
\lim _{t \rightarrow \infty} \frac{1}{t^{2}} \int_{t_{1}}^{t} \frac{1}{a(u)} \int_{t_{1}}^{u} g(s) d s d u<\infty,
$$

where the function $g(t)$ is defined as in (7) for any $t_{1} \geq c$. If $x$ is a positive solution of (1), then $\limsup _{t \rightarrow \infty}\left(x(t) / t^{2}\right)<\infty$.
Proof. Let $x(t)$ be an eventually positive solution of (1). We may assume that $x(t)>0$ for $t \geq t_{1}$ for some $t_{1} \geq c$. We let $F(t)=f(t, x(t))$. In view of (i) and (ii) we may then write

$$
\begin{aligned}
& \left(a(t) x^{\prime}(t)\right)^{\prime} \leq c_{0}+\frac{1}{\Gamma(\alpha)} \int_{c}^{t_{1}}(t-s)^{\alpha-1}|F(s)| d s \\
& +\frac{1}{\Gamma(\alpha)} \int_{c}^{t}(t-s)^{\alpha-1} e(s) d s+\frac{1}{\Gamma(\alpha)} \\
& \cdot \int_{t_{1}}^{t}(t-s)^{\alpha-1} s^{\gamma-1}\left[h(s) x^{\lambda}(s)-m(s) x(s)\right] d s \\
& +\frac{1}{\Gamma(\alpha)} \int_{t_{1}}^{t}(t-s)^{\alpha-1} s^{\gamma-1} m(s) x(s) d s .
\end{aligned}
$$

Applying (6) of Lemma 2 to $\left[h(s) x^{\lambda}(s)-m(s) x(s)\right]$ with

$$
\begin{aligned}
& X=(h)^{1 / \lambda} x \\
& Y=\left(\frac{1}{\lambda} m\left(h^{-1 / \lambda}\right)\right)^{1 /(\lambda-1)}
\end{aligned}
$$

we have

$$
\begin{aligned}
& h(s) x^{\lambda}(s)-m(s) x(s) \\
& \quad \leq(1-\lambda) \lambda^{\lambda /(1-\lambda)} m^{\lambda /(\lambda-1)}(s) h^{1 /(1-\lambda)}(s),
\end{aligned}
$$

and hence we obtain

$$
\begin{aligned}
& \left(a(t) x^{\prime}(t)\right)^{\prime} \leq c_{0}+\frac{1}{\Gamma(\alpha)} \int_{c}^{t_{1}}(t-s)^{\alpha-1}|F(s)| d s \\
& +\frac{1}{\Gamma(\alpha)} \int_{c}^{t}(t-s)^{\alpha-1} e(s) d s+\frac{\left((1-\lambda) \lambda^{\lambda /(1-\lambda)}\right)}{\Gamma(\alpha)} \\
& \cdot \int_{t_{1}}^{t}(t-s)^{\alpha-1} s^{\gamma-1}\left[m^{\lambda /(\lambda-1)}(s) h^{1 /(1-\lambda)}(s)\right] d s \\
& +\frac{1}{\Gamma(\alpha)} \int_{t_{1}}^{t}(t-s)^{\alpha-1} s^{\gamma-1} m(s) x(s) d s .
\end{aligned}
$$

Integrating inequality (15) from $t_{1}$ to $t$ and interchanging the order of integration, one can easily obtain

$$
\begin{gathered}
x^{\prime}(t) \leq \frac{a\left(t_{1}\right) x^{\prime}\left(t_{1}\right)}{a(t)}+\frac{c_{0}\left(t-t_{1}\right)}{a(t)}+\frac{1}{a(t)} \int_{t_{1}}^{t} g(s) d s \\
+\frac{1}{a(t) \Gamma(\alpha)} \int_{t_{1}}^{t} \int_{t_{1}}^{u}(u-s)^{\alpha-1} s^{\gamma-1} m(s) x(s) d s d u \\
+\frac{1}{a(t) \Gamma(\alpha)} \int_{t_{1}}^{t} \int_{c}^{t_{1}}\left(t_{1}-s\right)^{\alpha-1}|F(s)| d s d u \\
+\frac{1}{a(t) \Gamma(\alpha)} \int_{t_{1}}^{t} \int_{c}^{u}(u-s)^{\alpha-1} e(s) d s d u .
\end{gathered}
$$


Interchanging the order of integration in second integral, we have

$$
\begin{aligned}
x^{\prime}(t) \leq & \frac{a\left(t_{1}\right) x^{\prime}\left(t_{1}\right)}{a(t)}+\frac{c_{0}\left(t-t_{1}\right)}{a(t)}+\frac{1}{a(t)} \int_{t_{1}}^{t} g(s) d s \\
& +k \int_{t_{1}}^{t}(t-s)^{\alpha} s^{\gamma-1} m(s) x(s) d s \\
& +\frac{1}{a(t) \Gamma(\alpha)} \int_{t_{1}}^{t} \int_{c}^{t_{1}}\left(t_{1}-s\right)^{\alpha-1}|F(s)| d s d u \\
& +\frac{1}{a(t) \Gamma(\alpha)} \int_{t_{1}}^{t} \int_{c}^{u}(u-s)^{\alpha-1} e(s) d s d u,
\end{aligned}
$$

where $k$ is the upper bound of the function $1 / \alpha \Gamma(\alpha) a(t)$.

Integrating (17) from $t_{1}$ to $t$ and interchanging the order of integration in the last integral, we find

$x(t)$

$$
\begin{aligned}
& \leq x\left(t_{1}\right)+a\left(t_{1}\right) x^{\prime}\left(t_{1}\right)\left(\int_{t_{1}}^{t} \frac{1}{a(s)} d s\right) \\
& +\int_{t_{1}}^{t} \frac{c_{0}\left(s-t_{1}\right)}{a(s)} d s+\int_{t_{1}}^{t} \frac{1}{a(u)} \int_{t_{1}}^{u} g(s) d s d u \\
& +\frac{1}{\Gamma(\alpha)} \int_{t_{1}}^{t} \frac{1}{a(v)} \int_{t_{1}}^{v} \int_{c}^{t_{1}}\left(t_{1}-s\right)^{\alpha-1}|F(s)| d s d u d v \\
& +\frac{1}{\Gamma(\alpha)} \int_{c}^{t} \frac{1}{a(v)} \int_{t_{1}}^{v} \int_{c}^{u}(u-s)^{\alpha-1} e(s) d s d u d v \\
& +\frac{k}{\alpha+1}\left(\int_{t_{1}}^{t}(t-s)^{\alpha+1} m(s) s^{\gamma-1} x(s) d s\right) .
\end{aligned}
$$

Now, one can easily see that

$$
\begin{aligned}
& x(t) \\
& \leq x\left(t_{1}\right)+a\left(t_{1}\right) x^{\prime}\left(t_{1}\right)\left(\int_{t_{1}}^{t} \frac{1}{a(s)} d s\right) \\
& \quad+\int_{t_{1}}^{t} \frac{c_{0}\left(s-t_{1}\right)}{a(s)} d s \\
& +\frac{1}{\Gamma(\alpha)} \int_{t_{1}}^{t} \frac{1}{a(v)} \int_{t_{1}}^{v} \int_{c}^{t_{1}}\left(t_{1}-s\right)^{\alpha-1}|F(s)| d s d u d v \\
& +\frac{1}{\Gamma(\alpha)} \int_{c}^{t} \frac{1}{a(v)} \int_{t_{1}}^{v} \int_{c}^{u}(u-s)^{\alpha-1} e(s) d s d u d v \\
& +\int_{t_{1}}^{t} \frac{1}{a(u)} \int_{t_{1}}^{u} g(s) d s d u \\
& +k^{*}\left(\int_{1_{1}}^{t}(t-s)^{\alpha+1} s^{\gamma-1} m(s) x(s) d s\right),
\end{aligned}
$$

where $k^{*}=(k /(\alpha+1))$. From the hypotheses of the theorem, we see that

$$
\begin{aligned}
z(t) & :=\frac{x(t)}{t^{2}} \\
& \leq C+k^{*}\left(\int_{t_{1}}^{t}(t-s)^{\alpha-1} s^{\gamma-1} m(s) x(s) d s\right),
\end{aligned}
$$

with $C$ as the upper bound of the function

$$
\begin{aligned}
& \frac{1}{t^{2}}\left[x\left(t_{1}\right)+a\left(t_{1}\right) x^{\prime}\left(t_{1}\right)\left(\int_{t_{1}}^{t} \frac{1}{a(s)} d s\right)\right. \\
& +\int_{t_{1}}^{t} \frac{c_{0}\left(s-t_{1}\right)}{a(s)} d s \\
& +\frac{1}{\Gamma(\alpha)} \int_{c}^{t} \frac{1}{a(v)} \int_{t_{1}}^{v} \int_{c}^{u}(u-s)^{\alpha-1} e(s) d s d u d v \\
& +\frac{1}{\Gamma(\alpha)} \int_{t_{1}}^{t} \frac{1}{a(v)} \int_{t_{1}}^{v} \int_{c}^{t_{1}}\left(t_{1}-s\right)^{\alpha-1}|F(s)| d s d u d v \\
& \left.+\int_{t_{1}}^{t} \frac{1}{a(u)} \int_{t_{1}}^{u} g(s) d s d u\right] .
\end{aligned}
$$

Applying Holder's inequality and Lemma 1, we obtain

$$
\begin{aligned}
& \int_{t_{1}}^{t}(t-s)^{\alpha-1} s^{\gamma-1} m(s) x(s) d s \\
& \leq\left(\int_{t_{1}}^{t}(t-s)^{p(\alpha-1)} s^{p(\gamma-1)} d s\right)^{1 / p} \\
& \cdot\left(\int_{t_{1}}^{t} m^{q}(s) x^{q}(s) d s\right)^{1 / q} \\
& \leq\left(\int_{0}^{t}(t-s)^{p(\alpha-1)} s^{p(\gamma-1)} d s\right)^{1 / p} \\
& \cdot\left(\int_{t_{1}}^{t} m^{q}(s) x^{q}(s) d s\right)^{1 / q} \leq\left(B t^{\theta}\right)^{1 / p} \\
& \cdot\left(\int_{t_{1}}^{t} m^{q}(s) x^{q}(s) d s\right)^{1 / q},
\end{aligned}
$$

where $B=B[p(\gamma-1)+1, p(\alpha-1)+1]$ and $\theta=p(\alpha+\gamma-2)+1=0$ and so

$$
\begin{aligned}
& \int_{t_{1}}^{t}(t-s)^{\alpha-1} s^{\gamma-1} m(s) x(s) d s \\
& \quad \leq B^{1 / p}\left(\int_{t_{1}}^{t} m^{q}(s) x^{q}(s) d s\right)^{1 / q} .
\end{aligned}
$$


Thus, inequality (20) becomes

$$
\begin{aligned}
z(t) & :=\frac{x(t)}{t^{2}} \leq C+k^{*} B^{1 / p}\left(\int_{t_{1}}^{t} m^{q}(s) x^{q}(s) d s\right)^{1 / q} \\
& =C+k^{*} B^{1 / p}\left(\int_{t_{1}}^{t} s^{2 q} m^{q}(s)\left(\frac{x(s)}{s^{2}}\right)^{q} d s\right)^{1 / q} \\
& =C+k^{*} B^{1 / p}\left(\int_{t_{1}}^{t} s^{2 q} m^{q}(s) z(s)^{q} d s\right)^{1 / q} .
\end{aligned}
$$

Using this inequality and the elementary inequality

$$
(x+y)^{q} \leq 2^{q-1}\left(x^{q}+y^{q}\right), \quad x, y \geq 0, q>1,
$$

we obtain from (24)

$$
\begin{aligned}
& z^{q}(t) \\
& \quad \leq 2^{q-1}\left((1+C)^{q}+k^{q} B^{q / p} \int_{t_{1}}^{t} s^{2 q} m^{q}(s) z^{q}(s) d s\right) .
\end{aligned}
$$

If we denote $u(t)=z^{q}(t)$, that is, $z(t)=u^{1 / q}(t), P=2^{q-1}[(1+$ $C)^{q}$, and $Q=2^{q-1} k^{q} B^{q / p}$, then

$$
u(t) \leq P+Q \int_{t_{1}}^{t} s^{2 q} m^{q}(s) u(s) d s, \quad t \geq t_{1} \geq c .
$$

The conclusions follow from Gromwell's inequality and conclude that

$$
\limsup _{t \rightarrow \infty} \frac{x(t)}{t^{2}}<\infty
$$

This completes the proof.

Remark 4. Condition (10) can be replaced by

$$
\begin{aligned}
& \lim _{t \rightarrow \infty} \frac{1}{t^{2}} \int_{c}^{t} \frac{1}{a(v)} \int_{t_{1}}^{v} \int_{c}^{u}(u-s)^{\alpha-1}|e(s)| d s d u d v \\
& \quad<\infty
\end{aligned}
$$

and the result remains valid.

The following example is illustrative.

Example 5. Let $p>1,0<\alpha=1-1 / 2 p<1, \alpha=\gamma$, and $q=p /(p-1)$. Clearly

$$
\begin{aligned}
p(\alpha-1)+1 & =p(\gamma-1)+1=p\left(1-\frac{1}{2 p}-1\right)+1 \\
& =\frac{1}{2}>0 \\
\theta & =p(\alpha+\gamma-2)+1=0 .
\end{aligned}
$$

Let the functions $a(t)$ and $b(t)$ be as in (i) and (ii) with $b(t)$ being a bounded function and let $a(t)=e^{t}, e(t)=t e^{t} \sin t$, and $f(t, x)=t^{\gamma-1} h(t) x^{\lambda}$, where $0<\lambda<1$ and $h: R^{+} \rightarrow R^{+}$ is a continuous function with $h(t)=m(t)$,

$$
\begin{aligned}
& \int^{\infty}\left(s^{2} h(s)\right)^{q} d s<\infty \\
& \limsup _{t \rightarrow \infty} \frac{1}{t^{2}}\left(\int_{t_{1}}^{t} e^{-u} \int_{t_{1}}^{u}(u-s)^{\alpha-1} s^{\gamma-1} h(s) d s d u\right) \\
& \quad<\infty
\end{aligned}
$$

Condition (10) is also fulfilled. Thus, all conditions of Theorem 3 are satisfied and hence every positive solution $x$ of (1) satisfies limsup $\operatorname{sum}_{t \rightarrow \infty}\left(x(t) / t^{2}\right)<\infty$.

Next, we consider the fractional differential equation

$$
{ }^{C} D_{c}^{\alpha} y(t)=e(t)+f(t, x(t)), \quad c>1, \alpha \in(0,1),
$$

where $y(t)=x^{\prime}(t), c_{0}=y(c) / \Gamma(1)=y(c)$, and $c_{0}$ is a real constant.

Now we give sufficient conditions under which any positive solution $x$ of (32) satisfies $x(t)=O(t)$ as $t \rightarrow \infty$.

Theorem 6. Let $0<\lambda<1$ and suppose that $p>1, q=$ $p /(p-1), 0<\alpha<1$ and $\gamma=2-\alpha-1 / p, p(\alpha-1)+1>0$, and $p(\gamma-1)+1>0$,

$$
\int_{t_{1}}^{\infty}(\operatorname{sm}(s))^{q} d s<\infty
$$

$$
\begin{gathered}
\limsup _{t \rightarrow \infty} \frac{1}{t} \int_{t_{1}}^{t} \int_{c}^{u}(t-s)^{\alpha-1} e(s) d s d u<\infty, \\
\liminf _{t \rightarrow \infty} \frac{1}{t} \int_{t_{1}}^{t} \int_{c}^{u}(t-s)^{\alpha-1} e(s) d s d u>-\infty, \\
\lim _{t \rightarrow \infty} \frac{1}{t} \int_{t_{1}}^{t} g(s) d s<\infty, \\
g(t) \text { is defined as in (7), }
\end{gathered}
$$

for any $t_{1} \geq c$. If $x$ is a positive solution of (32), then $\lim \sup _{t \rightarrow \infty}(x(t) / t)<\infty$.

Proof. Let $x(t)$ be an eventually positive solution of (32). We may assume that $x(t)>0$ for $t \geq t_{1}$ for some $t_{1} \geq c$. We let $F(t)=f(t, x(t))$. In view of (ii) we may then write

$$
\begin{gathered}
x^{\prime}(t) \leq c_{0}+\frac{1}{\Gamma(\alpha)} \int_{c}^{t_{1}}(t-s)^{\alpha-1}|F(s)| d s+\frac{1}{\Gamma(\alpha)} \\
\cdot \int_{c}^{t}(t-s)^{\alpha-1} e(s) d s+\frac{1}{\Gamma(\alpha)} \\
\cdot \int_{t_{1}}^{t}(t-s)^{\alpha-1} s^{\gamma-1}\left[h(s) x^{\lambda}(s)-m(s) x(s)\right] d s \\
+\frac{1}{\Gamma(\alpha)} \int_{t_{1}}^{t}(t-s)^{\alpha-1} s^{\gamma-1} m(s) x(s) d s .
\end{gathered}
$$


Proceeding as in the proof of Theorem 3, we obtain

$$
\begin{gathered}
x(t)^{\prime} \leq c_{0}+\frac{1}{\Gamma(\alpha)} \int_{c}^{t_{1}}(t-s)^{\alpha-1}|F(s)| d s+\frac{1}{\Gamma(\alpha)} \\
\cdot \int_{c}^{t}(t-s)^{\alpha-1} e(s) d s+\frac{\left((1-\lambda) \lambda^{\lambda /(1-\lambda)}\right)}{\Gamma(\alpha)} \\
\cdot \int_{t_{1}}^{t}(t-s)^{\alpha-1} s^{\gamma-1}\left[m^{\lambda /(\lambda-1)}(s) h^{1 /(1-\lambda)}(s)\right] d s \\
+\frac{1}{\Gamma(\alpha)} \int_{t_{1}}^{t}(t-s)^{\alpha-1} s^{\gamma-1} m(s) x(s) d s .
\end{gathered}
$$

Integrating inequality (37) from $t_{1}$ to $t$ and interchanging the order of integration, one can easily obtain

$$
\begin{gathered}
x(t) \leq x\left(t_{1}\right)+c_{0}\left(t-t_{1}\right)+\int_{t_{1}}^{t} g(s) d s+\frac{1}{\Gamma(\alpha)} \\
\cdot \int_{t_{1}}^{t} \int_{t_{1}}^{u}(u-s)^{\alpha-1} s^{\gamma-1} m(s) x(s) d s d u \frac{1}{\Gamma(\alpha)} \\
\cdot \int_{t_{1}}^{t} \int_{c}^{t_{1}}\left(t_{1}-s\right)^{\alpha-1}|F(s)| d s d u+\frac{1}{\Gamma(\alpha)} \\
\cdot \int_{t_{1}}^{t} \int_{c}^{u}(t-s)^{\alpha-1} e(s) d s d u .
\end{gathered}
$$

Interchanging the order of integration in second integral we have

$$
\begin{aligned}
x(t) \leq & x\left(t_{1}\right)+c_{0}\left(t-t_{1}\right)+\int_{t_{1}}^{t} g(s) d s \\
& +\frac{t}{\alpha \Gamma(\alpha)} \int_{t_{1}}^{t}(t-s)^{\alpha-1} s^{\gamma-1} m(s) x(s) d s \\
& +\frac{1}{\Gamma(\alpha)} \int_{t_{1}}^{t} \int_{c}^{t_{1}}\left(t_{1}-s\right)^{\alpha-1}|F(s)| d s d u \\
& +\frac{1}{\Gamma(\alpha)} \int_{t_{1}}^{t} \int_{c}^{u}(t-s)^{\alpha-1} e(s) d s d u .
\end{aligned}
$$

The rest of the proof is similar to that of Theorem 3 and hence is omitted.

Example 7. Let $p>1,0<\alpha=1-1 / 2 p<1, \alpha=\gamma$, and $q=p /(p-1)$. Clearly

$$
\begin{aligned}
p(\alpha-1)+1 & =p(\gamma-1)+1=p\left(1-\frac{1}{2 p}-1\right)+1 \\
& =\frac{1}{2}>0, \\
\theta & =p(\alpha+\gamma-2)+1=0 .
\end{aligned}
$$

Let the functions $e(t)=t \sin t$ and $f(t, x)=t^{\gamma-1} h(t) x^{\lambda}$, where $0<\lambda<1$ and $h: R^{+} \rightarrow R^{+}$is a continuous function with $h(t)=m(t)$,

$$
\begin{array}{r}
\int^{\infty}(\operatorname{sh}(s))^{q} d s<\infty, \\
\limsup _{t \rightarrow \infty} \frac{1}{t}\left(\int_{t_{1}}^{u}(u-s)^{\alpha-1} s^{\gamma-1} h(s) d s\right)<\infty .
\end{array}
$$

Condition (34) is also fulfilled. Thus, all conditions of Theorem 8 are satisfied and hence every positive solution $x$ of (32) satisfies $\lim \sup _{t \rightarrow \infty}(x(t) / t)<\infty$.

Finally, we consider the fractional differential equation

$$
{ }^{C} D_{c}^{\alpha} x(t)=e(t)+f(t, x(t)), \quad c>1, \alpha \in(0,1),
$$

where $x(c)=c_{0}$ and $c_{0}$ is a real constant.

Now, we give sufficient conditions for the boundedness of any positive solution of (42).

Theorem 8. Let $0<\lambda<1$ and suppose that $p>1, q=$ $p /(p-1), 0<\alpha<1$ and $\gamma=2-\alpha-1 / p, p(\alpha-1)+1>0$, and $p(\gamma-1)+1>0$,

$$
\int_{t_{1}}^{\infty} m^{q}(s) d s<\infty,
$$

$$
\begin{aligned}
& \limsup _{t \rightarrow \infty} \int_{t_{1}}^{t}(t-s)^{\alpha-1} e(s) d s<\infty, \\
& \liminf _{t \rightarrow \infty} \int_{t_{1}}^{t}(t-s)^{\alpha-1} e(s) d s>-\infty, \\
& \lim _{t \rightarrow \infty} g(t)<\infty, \\
& g(t) \text { is defined as in (7), }
\end{aligned}
$$

for any $t_{1} \geq c$. If $x$ is a positive solution of (42), then $x(t)$ is bounded.

Proof. Let $x(t)$ be an eventually positive solution of (42). We may assume that $x(t)>0$ for $t \geq t_{1}$ for some $t_{1} \geq c$. We let $F(t)=f(t, x(t))$. In view of (ii) we may then write

$$
\begin{gathered}
x(t) \leq c_{0}+\frac{1}{\Gamma(\alpha)} \int_{c}^{t_{1}}(t-s)^{\alpha-1}|F(s)| d s+\frac{1}{\Gamma(\alpha)} \\
\cdot \int_{c}^{t}(t-s)^{\alpha-1} e(s) d s+\frac{1}{\Gamma(\alpha)} \\
\cdot \int_{t_{1}}^{t}(t-s)^{\alpha-1} s^{\gamma-1}\left[h(s) x^{\lambda}(s)-m(s) x(s)\right] d s \\
+\frac{1}{\Gamma(\alpha)} \int_{t_{1}}^{t}(t-s)^{\alpha-1} s^{\gamma-1} m(s) x(s) d s .
\end{gathered}
$$


Proceeding as in the proof of Theorem 3, we obtain

$$
\begin{gathered}
x(t) \leq c_{0}+\frac{1}{\Gamma(\alpha)} \int_{c}^{t_{1}}(t-s)^{\alpha-1}|F(s)| d s+\frac{1}{\Gamma(\alpha)} \\
\cdot \int_{c}^{t}(t-s)^{\alpha-1} e(s) d s+\frac{\left((1-\lambda) \lambda^{\lambda /(1-\lambda)}\right)}{\Gamma(\alpha)} \\
\cdot \int_{t_{1}}^{t}(t-s)^{\alpha-1} s^{\gamma-1}\left[m^{\lambda /(\lambda-1)}(s) h^{1 /(1-\lambda)}(s)\right] d s \\
+\frac{1}{\Gamma(\alpha)} \int_{t_{1}}^{t}(t-s)^{\alpha-1} s^{\gamma-1} m(s) x(s) d s
\end{gathered}
$$

or

$$
\begin{aligned}
x(t) \leq c_{0} & +\frac{1}{\Gamma(\alpha)} \int_{c}^{t_{1}}(t-s)^{\alpha-1}|F(s)| d s \\
& +\frac{1}{\Gamma(\alpha)} \int_{c}^{t}(t-s)^{\alpha-1} e(s) d s+g(t) \\
& +\frac{1}{\Gamma(\alpha)} \int_{t_{1}}^{t}(t-s)^{\alpha-1} s^{\gamma-1} m(s) x(s) d s .
\end{aligned}
$$

The rest of the proof is similar to that of Theorem 3 and hence is omitted.

Example 9. Let $p>1,0<\alpha=1-1 / 2 p<1, \alpha=\gamma$, and $q=p /(p-1)$. Clearly

$$
\begin{aligned}
p(\alpha-1)+1 & =p(\gamma-1)+1=p\left(1-\frac{1}{2 p}-1\right)+1 \\
& =\frac{1}{2}>0 \\
\theta & =p(\alpha+\gamma-2)+1=0 .
\end{aligned}
$$

Let the functions $e(t)=\sin t$ and $f(t, x)=t^{\gamma-1} h(t) x^{\lambda}$, where $0<\lambda<1$ and $h: R^{+} \rightarrow R^{+}$is a continuous function with $h(t)=m(t)$,

$$
\begin{array}{r}
\int^{\infty} h^{q}(s) d s<\infty, \\
\limsup _{t \rightarrow \infty}\left(\int_{t_{1}}^{t}(t-s)^{\alpha-1} s^{\gamma-1} h(s) d s\right)<\infty .
\end{array}
$$

Condition (34) is also fulfilled. Thus, all conditions of Theorem 8 are satisfied and hence every positive solution of (42) is bounded.

Similar reasoning to that in the sublinear case guarantees the following theorems for the integrodifferential equation (1) when $\lambda=1$.

Theorem 10. Let $\lambda=1$ and the hypotheses of Theorems 3-8 hold with $m(t)=h(t)$.

Then the conclusion of Theorems 3-8 holds.

\section{General Remarks}

(1) The results of this paper are presented in a form which is essentially new and it can also be obtained for higher fractional differential equations (1) of order $\alpha \in(n-1, n), n \geq 1$.

(2) It would be of interest to study (1) when $f$ satisfies condition (ii) with $\lambda>1$.

\section{Conflict of Interests}

The author declares that they have no competing interests.

\section{Acknowledgments}

The author is extremely grateful to the reviewers and the handling editor for many helpful comments and suggestions, which have contributed much to the improvement of this paper.

\section{References}

[1] D. Baleanu, J. A. T. Machado, and A. C.-J. Luo, Fractional Dynamics and Control, Springer, 2012.

[2] V. Lakshmikantham, S. Leela, and J. V. Devi, Theory of Fractional Dynamic Systems, Cambridge Scientific Publishers, 2009.

[3] A. A. Kilbas, H. M. Srivastava, and J. J. Trujillo, Theory and Applications of Fractional Differential Equations, vol. 204 of North-Holland Mathematics Studies, Elsevier, Amsterdam, The Netherlands, 2006.

[4] K. S. Miller and B. Ross, An Introduction to the Fractional Calculus and Differential Equations, John Wiley \& Sons, New York, NY, USA, 1993.

[5] Q.-H. Ma, J. Pečarić, and J.-M. Zhang, "Integral inequalities of systems and the estimate for solutions of certain nonlinear twodimensional fractional differential systems," Computers and Mathematics with Applications, vol. 61, no. 11, pp. 3258-3267, 2011.

[6] I. Podlubny, Fractional Differential Equations, Academic Press, San Diego, Calif, USA, 1999.

[7] A. P. Prudnikov, Zu. A. Brychkov, and O. I. Marichev, Integral and Series, vol. 1 of Elementary Functions, Nauka, Moscow, Russia, 1981 (Russian).

[8] S. G. Samko, A. A. Kilbas, and O. I. Marichev, Fractional Integrals and Derivatives; Theory and Applications, Gordon and Breach Science Publishers, New York, NY, USA, 1993.

[9] M. Bohner, S. Grace, and N. Sultana, "Asymptotic behavior of nonoscillatory solutions of higher-order integro-dynamic equations," Opuscula Mathematica, vol. 34, no. 1, pp. 5-14, 2014.

[10] S. R. Grace and A. Zafer, "Oscillatory behavior of integrodynamic and integral equations on time scales," Applied Mathematics Letters, vol. 28, pp. 47-52, 2014.

[11] S. R. Grace, J. R. Graef, and A. Zafer, "Oscillation of integrodynamic equations on time scales," Applied Mathematics Letters, vol. 26, no. 4, pp. 383-386, 2013.

[12] S. R. Grace, J. R. Graef, S. Panigrahi, and E. Tunc, "On the oscillatory behavior of Volterra integral equations on timescales," Panamerican Mathematical Journal, vol. 23, no. 2, pp. 35-41, 2013. 
[13] S. R. Grace, R. P. Agarwal, P. J. Y. Wong, and A. Zafer, "On the oscillation of fractional differential equations," Fractional Calculus and Applied Analysis, vol. 15, no. 2, pp. 222-231, 2012.

[14] M. Caputo, "Linear models of dissipation whose Q is almost frequency independent II," Geophysical Journal of the Royal Astronomical Society, vol. 13, pp. 529-535, 1967.

[15] K. Diethelm, The Analysis of Fractional Differential Equations, Lecture Notes in Mathematics, Springer, Berlin, Germany, 2010.

[16] K. M. Furati and N.-E. Tatar, "Power-type estimates for a nonlinear fractional differential equation," Nonlinear Analysis. Theory, Methods \& Applications, vol. 62, no. 6, pp. 1025-1036, 2005.

[17] G. H. Hardy, I. E. Littlewood, and G. Polya, Inequalities, Cambridge University Press, 1959. 


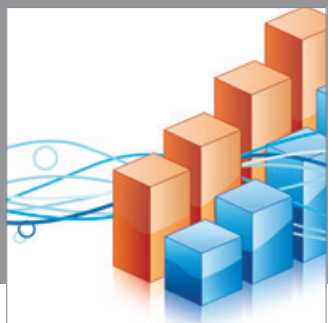

Advances in

Operations Research

mansans

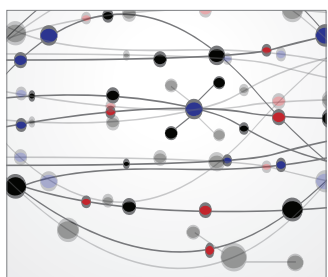

The Scientific World Journal
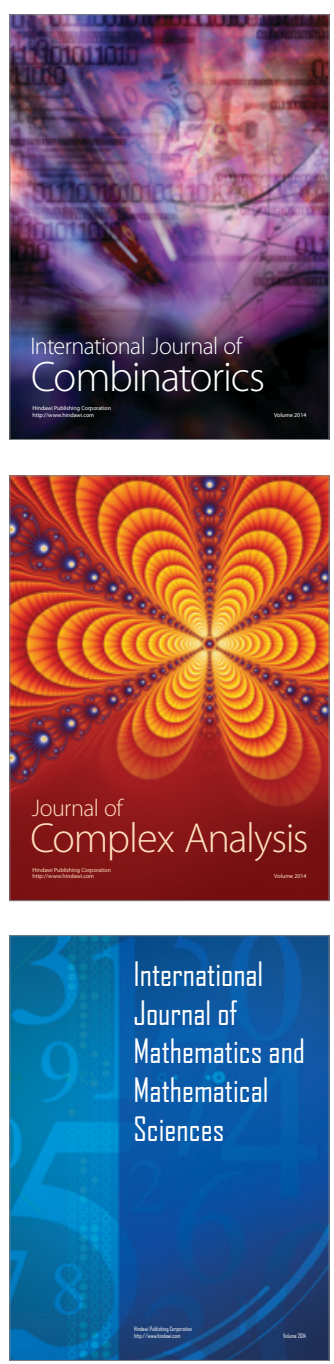
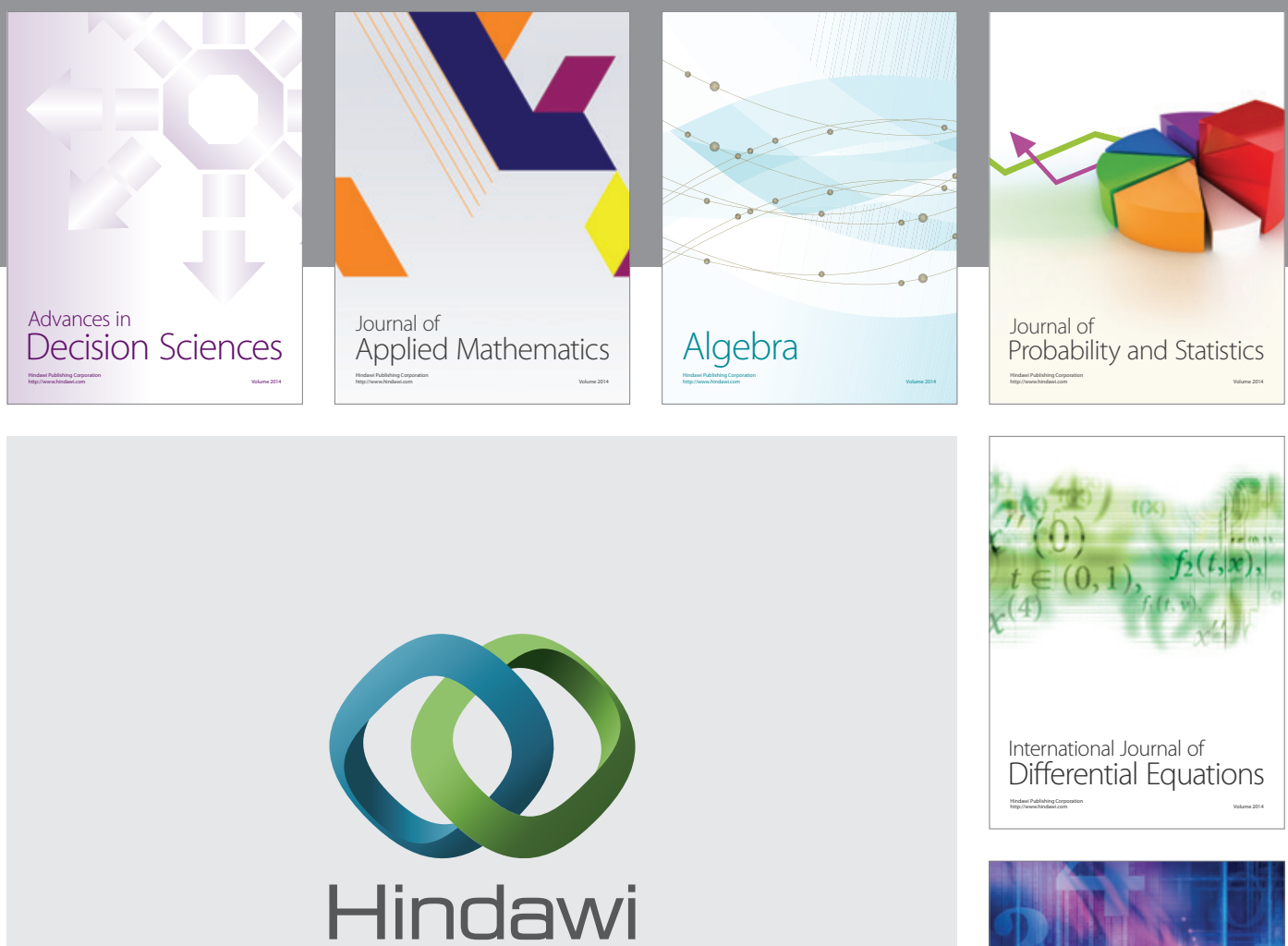

Submit your manuscripts at http://www.hindawi.com
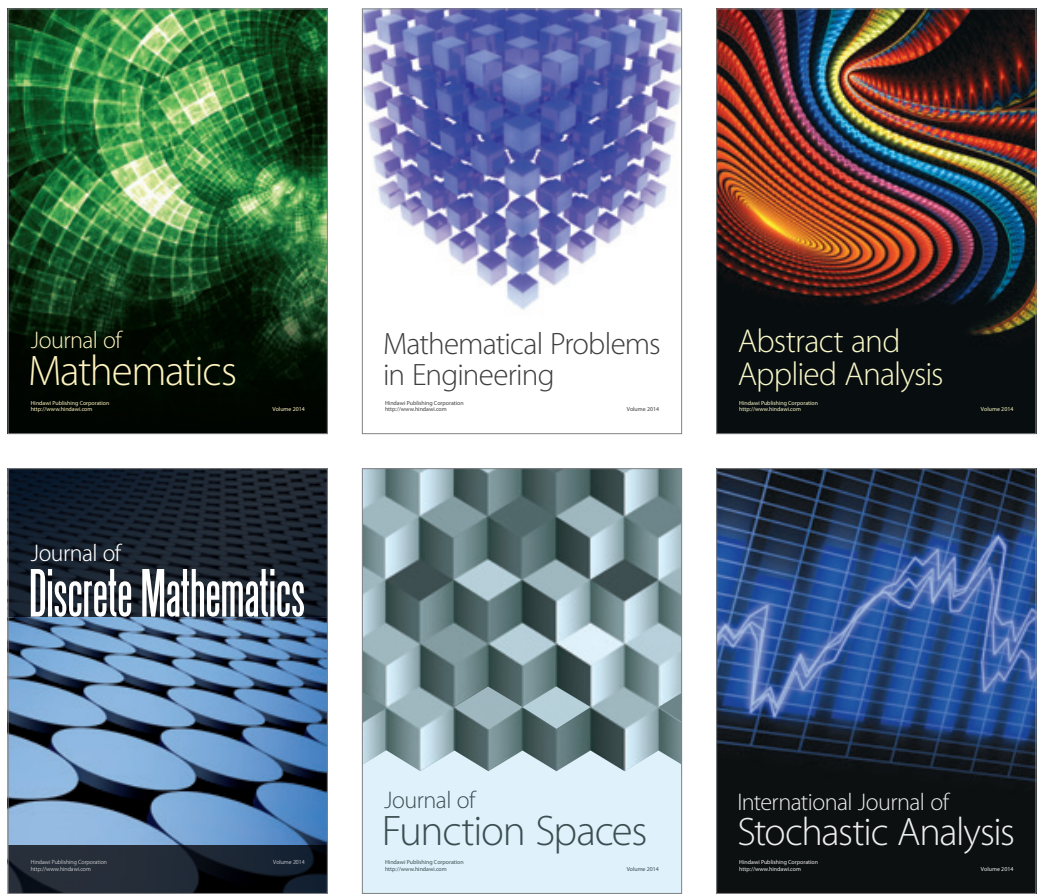

Journal of

Function Spaces

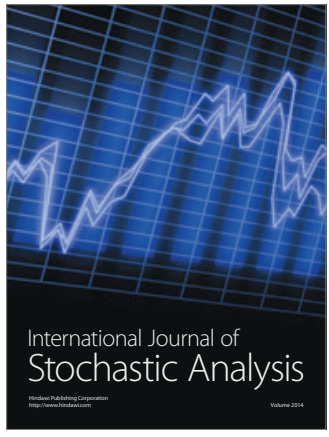

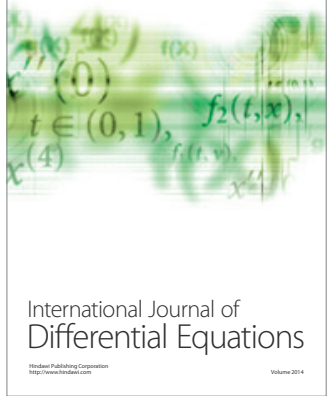
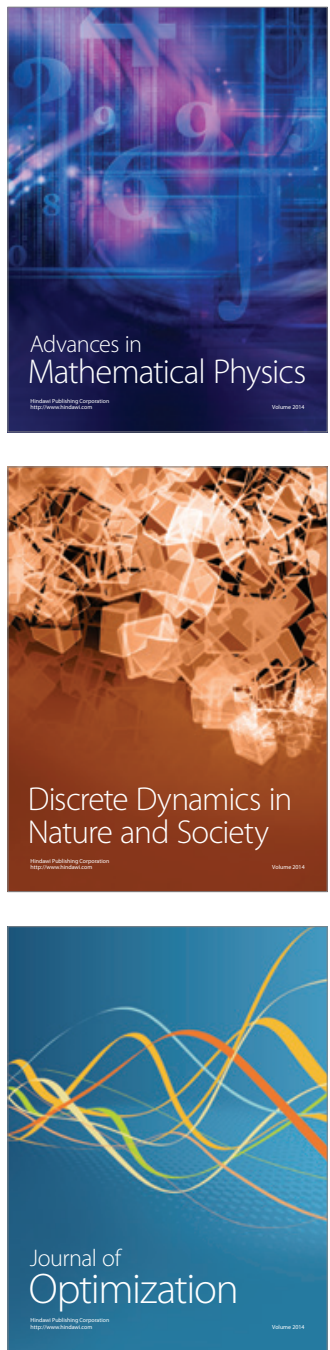